\title{
Separation and Detection of Cyanine-Labeled Amino Acids by Micellar Electrokinetic Chromatography Combined with Fluorescence Detection Using Diode-Based Solid-State Lasers
}

\author{
Takashi KaneTA, Takeshi Komatsubara, Hiroki ShIBA and Totaro IMASAKA ${ }^{\dagger}$ \\ Department of Chemical Science and Technology, Faculty of Engineering, Kyushu University, \\ Hakozaki, Fukuoka 812-8581, Japan
}

\begin{abstract}
Keywords Micellar electrokinetic chromatography, laser-induced fluorescence detection, diode laser, diode laserpumped YAG laser, amino acid
\end{abstract}

Laser-induced fluorescence (LIF) detection is one of the most sensitive detection techniques available for capillary electrophoresis (CE) because of the small required detection volume. There have been several reports on CE combined with LIF, the determination of amino acids ${ }^{1-3}$, DNAs ${ }^{4,5}$, and polycyclic aromatic hydrocarbons. ${ }^{6-8}$ However, most lasers have several limitations, including cost, size, complex maintenance, and a short usable lifetime. Diode lasers are used to solve these problems. We have reported on analytical applications using diode lasers as excitation sources for $\mathrm{CE}$ and micellar electrokinetic chromatography (MEKC) LIF detection of amino acids ${ }^{9,10}$, polycyclic aromatic hydrocarbons $\mathrm{s}^{11,12}$, and oligonucleotides. ${ }^{13}$

One significant limitation of diode lasers as LIF excitation sources is that the laser emission wavelength is too long, and it is difficult to obtain chemical reagents which have absorption bands at such long wavelengths. We have demonstrated that the second-harmonic wave of a diode laser (415 nm) could be used to determine polycyclic aromatic hydrocarbons. ${ }^{11}$ However, the power of the laser $(10 \mu \mathrm{W})$ was not large enough for it to be used as an excitation light source for many LIF applications.

A diode laser emitting at $635 \mathrm{~nm}$ is commercially available. Furthermore, a diode laser-pumped YAG laser emitting at $532 \mathrm{~nm}$, which is the second-harmonic wave, is also available. The power of these lasers is large (several $\mathrm{mW}$ ) enough to use for LIF detection. Also, labeling reagents suitable for diode laser excitation, such as thionine derivatives and cyanine derivatives, are now available. These compounds are useful for applying diode LIF to the determination of several compounds.

In this paper, a diode laser and diode laser excited YAG laser were used as excitation sources in LIF to determine amino acids labeled by cyanine derivatives.

† To whom correspondence should be addressed.
Two kinds of cyanine derivatives, which have absorption maxima at 550 and $649 \mathrm{~nm}$, respectively, were employed to label amino acids. Cyanine-labeled amino acids were separated by MEKC and detected by LIF based on diode laser excitation.

\section{Experimental}

\section{Apparatus}

The capillary electrophoretic apparatus is described elsewhere. ${ }^{14}$ A diode laser emitting at $635 \mathrm{~nm}$ (ILEE Laser Innovation, Urdorf, Switzerland; Model LDA1001, output power $<5 \mathrm{~mW}$ ) and diode laser-excited YAG laser emitting at $532 \mathrm{~nm}$ (Tokyo Instruments, Tokyo, Japan; Model LL-01 No. 1941, output power, 1 $-10 \mathrm{~mW}$ ) are used as excitation sources. The laser beam is focused by a microscope objective lens into a capillary (GL Sciences Inc., Tokyo, Japan; $50 \mu \mathrm{m}$ i.d., $375 \mu \mathrm{m}$ o.d.). Fluorescence is collected by an objective lens and passed through interference and spatial filters. The signal from a photomultiplier tube is measured by a strip-chart recorder. The sample is injected by gravity. A high-voltage power supply, Model HCZE30PN0.25 (Matsusada Precision Devices, Shiga, Japan), is used for applying voltage.

\section{Chemicals}

Cyanine derivatives for labeling amino acids, Cy3 and Cy5 (FluoroLink Mono Reactive Dye), were obtained from Amersham (Tokyo, Japan). The structures of the labeling reagents are shown in Fig. 1. Glycine, alanine, aspartic acid, and glutamic acid were purchased from Kishida Chemical Co. (Tokyo, Japan). All other chemicals were obtained from Wako Pure Chemical (Osaka, Japan).

Amino acids were labeled with cyanine derivatives as follows: after $1 \mathrm{mg}$ of the cyanine derivatives was dissolved in freshly distilled dimethylformamide, $10 \mu \mathrm{l}$ of 
<smiles></smiles><smiles></smiles>

Cy5

Fig. 1 Structures of cyanine derivatives.

the solution was added to each amino acid solution containing phosphate or carbonate buffer $(\mathrm{pH} 9)$. After standing for $1 \mathrm{~h}$ at room temperature, the solutions were mixed and diluted. The concentration of the mixture was adjusted to $10 \mu \mathrm{M}$ for each amino acid.

\section{Results and Discussion}

In preliminary studies, the separation of cyaninelabeled amino acids was carried out in the conventional CZE mode. However, the resolution between the amino acids was poor, because of small differences in the electrophoretic mobilities between the cyaninelabeled amino acids. The cyanine derivatives used in this study have a large molecular weight $\left(654.7 \mathrm{~g} \mathrm{~mol}^{-1}\right.$ for $\mathrm{Cy} 3$ and $792.0 \mathrm{~g} \mathrm{~mol}^{-1}$ for Cy5) and a negative charge, as shown in Fig. 1. Thus, the electrophoretic mobilities of amino acids is changed by tagging with cyanine dyes. The difference in the mobilities of amino acids might be reduced by tagging with cyanine dyes since the labeling reagents have a negative charge. Therefore, separation of the amino acids was attempted using MEKC.

The separation of Cy3-labeled amino acids is shown in Fig. 2. To separate Cy3-labeled amino acids, $\beta$ cyclodextrin was used as a modifier. The separation of seven amino acids was achieved using a running buffer containing $50 \mathrm{mM}$ sodium dodecylsulfate and $10 \mathrm{mM}$ $\beta$-cyclodextrin. Also, the separation of Cy5-labeled amino acids is shown in Fig. 3. Nine amino acids were separated (Fig. 3) without the addition of $\beta$-CD. The difference between the separations of Cy3- and Cy5labeled amino acids is explained as follows: $\mathrm{Cy} 5$ has a negative charge, as does Cy3. Thus, the change in the electrophoretic mobilities of Cy5-labeled amino acids

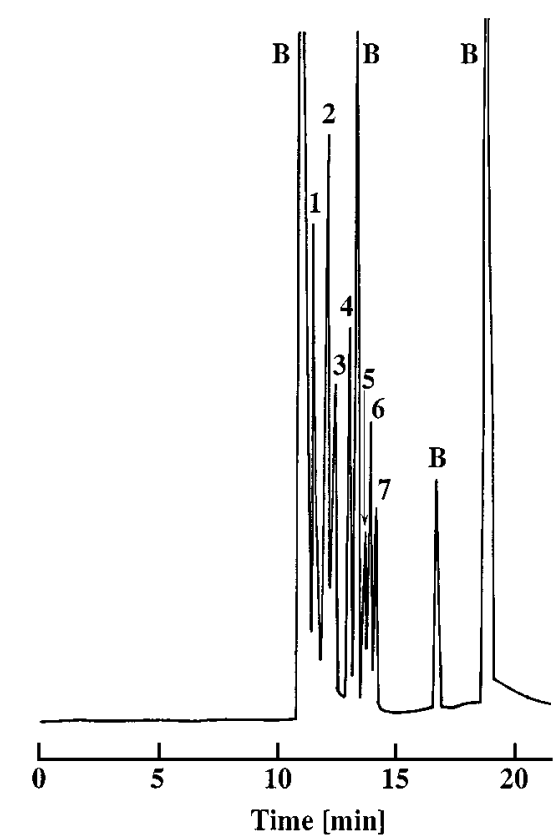

Fig. 2 Chromatogram of Cy3-labeled amino acids. (1) glycine, (2) histidine, (3) leucine, (4) phenylalanine, (5) glutamic acid, (6) aspartic acid, (7) tryptophan, (B) blank. Buffer, $10 \mathrm{mM}$ borate ( $\mathrm{pH} 9.0$ ) containing $50 \mathrm{mM}$ SDS and $10 \mathrm{mM} \beta$-CD; applied voltage, $15 \mathrm{kV}(8 \mu \mathrm{A})$; capillary, total length $=60 \mathrm{~cm}$, effective length $=50 \mathrm{~cm}$; sample concentration, $10 \mu \mathrm{M}$ for each amino acid.

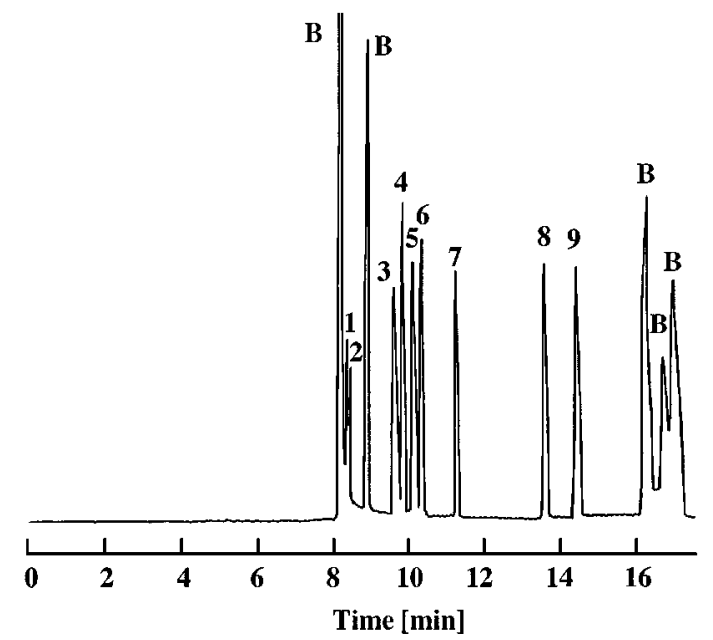

Fig. 3 Chromatogram of Cy5-labeled amino acids. (1) aspartic acid, (2) alanine, (3) glycine, (4) glutamic acid, (5) tyrosine, (6) valine, (7) histidine, (8) phenylalanine, (9) tryptophan, (B) blank. Buffer, $10 \mathrm{mM}$ phosphate (pH 9.2) containing $50 \mathrm{mM} \mathrm{SDS}$; applied voltage, $18 \mathrm{kV}(20 \mu \mathrm{A})$; capillary, total length $=60 \mathrm{~cm}$, effective length $=50 \mathrm{~cm}$; sample concentration, $10 \mu \mathrm{M}$ for each amino acid.

should be similar to that of Cy3-labeled amino acids. However, since Cy5 has a longer polymethine chain than does $\mathrm{Cy} 3, \mathrm{Cy} 5$ should be retained more effectively onto/into SDS micelles than Cy3. Consequently, Cy5labeled amino acids were separated using a $50 \mathrm{mM}$ SDS solution without $\beta$-CD. 
The detection limits, which were calculated for $S / N=3$ from Figs. 2 and 3, were $4.2 \times 10^{-8}$ for Cy3-labeled tryptophan and $6.1 \times 10^{-8} \mathrm{M}$ for Cy5-labeled tryptophan. Thus, it is considered that there is no difference between the labeling efficiency for $\mathrm{Cy} 3$ and $\mathrm{Cy} 5$. In this study, color filters are used to remove scattered light from the capillary wall. However, to achieve more sensitive detection, band pass filters or notch filters, which can remove the laser light more efficiently, must be employed. Furthermore, a detection system based on a method using a sheath flow is effective to improve the detection limit, as demonstrated in our previous paper ${ }^{15}$, in which an improvement of seven orders of magnitude in the detection limit was achieved.

In conclusion, the detection of cyanine-labeled amino acids was demonstrated by LIF using small solid-state lasers, such as a diode laser and diode laser-pumped YAG laser. These lasers have a sufficiently large power to use as an excitation source of LIF detection in CE. Two different cyanine derivatives, Cy3 and Cy5, were used for the diode laser and the YAG laser excitation, respectively. The detection limit for $\mathrm{Cy} 3$ was similar to that for Cy5. Thus, these lasers should be useful for the determination of amino acids. Furthermore, some cyanine derivatives, the excitation maxima of which are in the deep-red region, are commercially available. Thus, it is expected that diode LIF will be applied to the determination of biological samples and DNA sequencing.

This research was supported by Grants-in-Aid for Scientific Research from the Ministry of Education of Japan. T. K. thanks Dr. Michael R. Shortreed for helpful discussions.

\section{References}

1. E. J. Gassmann, J. Kuo and R. N. Zare, Science [Washington, D.C.], 222, 1298 (1985).

2. J. Liu, Y.-Z. Hsieh, D. Wiesler and M. Novotny, Anal. Chem., 63, 408 (1991).

3. S. Wu and N. J. Dovichi, Talanta, 39, 173 (1992).

4. H. E. Schwartz and K. J. Ulfelder, Anal. Chem., 64, 1737 (1992).

5. M. C. Ruiz-Martinez, J. Berka, A. Belenkii, F. Foret, A. W. Miller and B. L. Karger, Anal. Chem., 65, 2851 (1993).

6. T. Imasaka, K. Nishitani and N. Ishibashi, Anal. Chim. Acta, 256, 3 (1992).

7. S. Nie, R. Dadoo and R. N. Zare, Anal. Chem., 65, 3571 (1993).

8. O. H. J. Szolar, R. S. Brown and J. H. Luong, Anal. Chem., 67, 3004 (1995).

9. T. Higashijima, T. Fuchigami, T. Imasaka and N. Ishibashi, Anal. Chem., 64, 711 (1992).

10. T. Fuchigami, T. Imasaka and M. Shiga, Anal. Chim. Acta, 282, 209 (1993).

11. T. Imasaka, K. Nishitani and N. Ishibashi, Analyst [London], 116, 1407 (1991).

12. T. Kaneta, T. Yamashita and T. Imasaka, Anal. Chim. Acta, 299, 371 (1995).

13. T. Kaneta, T. Okamoto and T. Imasaka, Anal. Sci., 12, 875 (1996).

14. T. Kaneta and T. Imasaka, Anal. Chem., 67, 829 (1995).

15. T. Fuchigami, T. Imasaka and M. Shiga, Anal. Chim. Acta, 282, 209 (1993).

(Received April 23, 1998)

(Accepted June 29, 1998) 